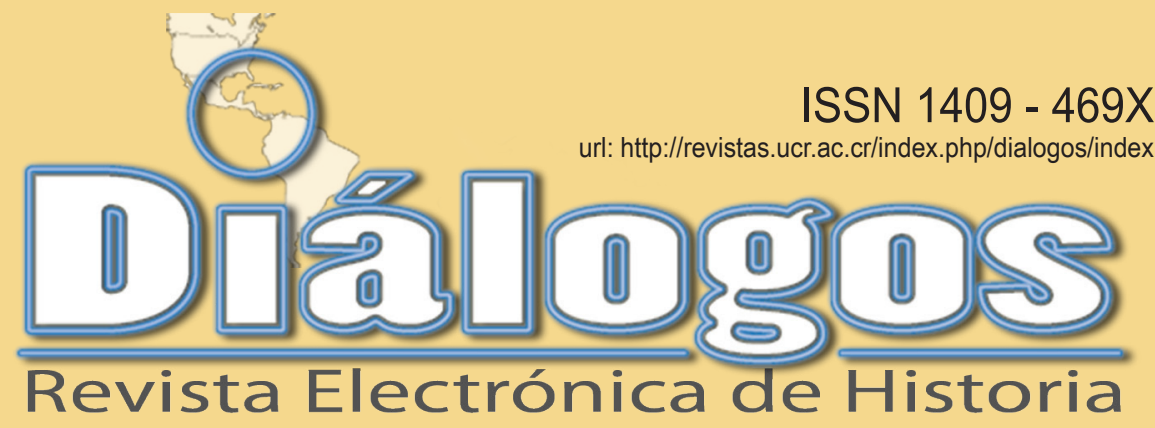

Escuela de Historia. Universidad de Costa Rica Volumen 15 Especial Región Occidente de Costa Rica - Octubre, 2014

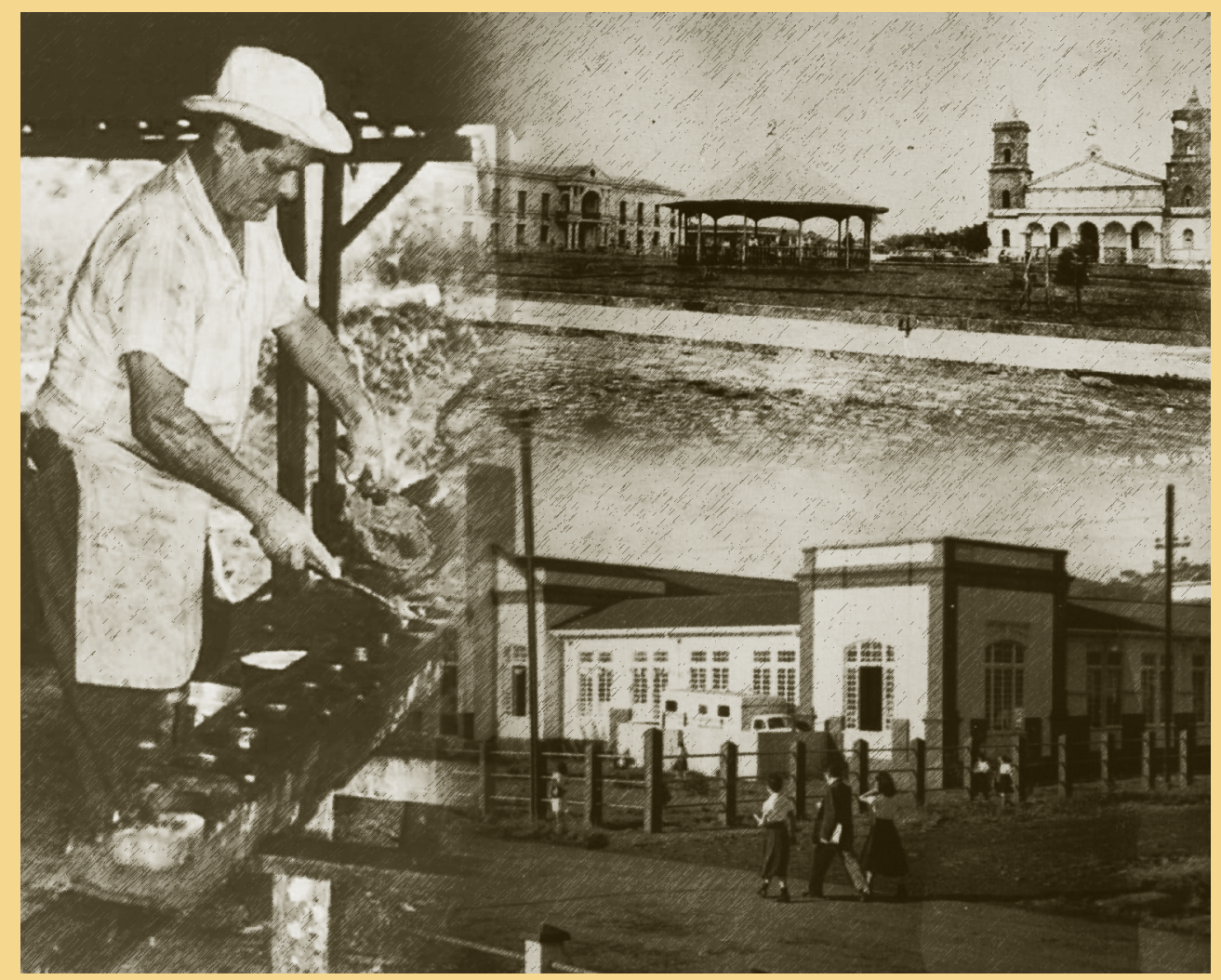

Director de la Revista: Dr. Juan José Marín Hernández juan.marinhernandez@ucr.ac.cr

Editor académico: Dr. David Díaz Arias - david.diaz@ucr.ac.cr

Editores invitados: M.Sc. William Solórzano Vargas - william.solorzano@ucr.ac.cr M.Ed. Maynor Badilla Vargas - mainor.badilla@ucr.ac.cr

Editora técnica: M.Sc. Marcela Quirós G. - marcela.quiros@ucr.ac.cr 
Dr. Juan José Marín Hernández, Catedrático. Director del Centro de Investigaciones Históricas de América Central. Universidad de Costa Rica. Costa Rica. juan. marin@ucr.ac.cr

Dr. David Díaz Arias: Catedrático. Historia Política, Director del posgrado de Historia y Docente de la Escuela de Historia, Universidad de Costa Rica, Costa Rica.david.diaz@ucr.ac.cr

Dr. Ronny Viales Hurtado. Catedrático. Historia Económica y Social. Universidad de Costa Rica. Director de la Escuela de Historia. Costa Rica. ronny. viales@ucr.ac.cr

MSc. Francisco Enríquez. Historia Social. Universidad de Costa Rica. Costa Rica. francisco.enriquez@ucr. ac.cr

Dra. Ana María Botey. Historia de los movimientos sociales. Universidad de Costa Rica. Costa Rica. abotey@gmail.com

\section{Miembros del Consejo Asesor Internacional:}

Dr. José Cal Montoya. Universidad de San Carlos de Guatemala. Guatemala. jecalm@correo.url.edu.gt

Dr. Juan Manuel Palacio. Universidad Nacional de San Martín. Argentina.jpalacio@unsam.edu.ar

Dr. Eduardo Rey. Universidad de Santiago de Compostela. España. ereyt@usc.es

Dr. Heriberto Cairo Carou. Departamento de Ciencia Política y de la Administración III - Universidad Complutense de Madrid. España. hcairoca@cps.ucm.es

Dra. Rosa de la Fuente. Departamento de Ciencia Política y de la Administración III Universidad Complutense de Madrid. España. rdelafuente@cps. ucm.es

Dr. Javier Franzé. Departamento de Ciencia Política y de la Administración III Universidad Complutense de Madrid. España. javier.franze@cps.ucm.es

Dr. Jaime Preciado Coronado Departamento de Estudios Ibéricos y Latinoamericanos. Universidad de Guadalajara. México.japreco@hotmail.com

Dr. Gerónimo de Sierra. Vicerrector de la Universidade Federal da Integração Latino-Americana (UNILA) y Departamento de Sociología, Facultad de Ciencias

Sociales de la Universidad de la República. Uruguay. geronimo@fcs.edu.uy

Dr. Antonio Palazuelos. Departamento de Ciencia Política y de la Administración III - Universidad Complutense de Madrid. España. palazuelosa@cps. ucm.es

Dr. Werner Mackenbach. Universidad Potsdam. Alemania.werner.mackenbach@uni-potsdam.de

Dr. Guillermo Castro. Ciudad del Saber Panamá. Panamá.gcastro@cdspanama.org

Dra. Natalia Milanesio. University of Houston. Estados Unidos.nmilane2@Central.UH.EDU

Dr. Ricardo González Leandri. Consejo Superior de Investigaciones Científicas - España. España. rgleandri@gmail.com

Dra. Mayra Espina. Centro de Estudios Psicológicos y Sociológicos, La Habana. Cuba.mjdcips@ceniai.inf.cu

Dra. Montserrat Llonch. Departamento de Economía e Historia Económica Universidad Autónoma de Barcelona. España. montserrat.llonch@uab.es

Dra. Estela Grassi. Universidad de Buenos Aires. Argentina. estelagrassi@gmail.com

Dra. Yolanda Blasco. Universidad de Barcelona. España. yolandablasco@ub.edu

Dr. Alfredo Falero. Departamento de Sociología. Universidad de la República. Uruguay. alfredof@adinet. com.uy

Portada:

Fotografía: ( Collage "Campesinos en la producción de dulce en un trapiche de Rincón de Mora, Ran Ramón, 1987" ; "Panorámica del Antiguo Palacio Municipal, Antigua Iglesia, Parque y Kiosco de San Ramón, hacia 1920; y Escuela Jorge Washinton, San Ramón hacia 1955). Estas fotografías pertenecen a la Colección Fotográfica del Museo Regional de San Ramón- UCR. Montaje fotográfico: Lic. Juan Gabriel Madrigal Cubero ).

\section{Equipo Técnico Editorial:}

Diagramación y

Edición técnica:

M.Sc. Marcela Quirós Garita. marcela.quiros@ucr.ac.cr

Soporte técnico: Kevin Trejos Vargas

Revisión filológica: Baruc Chavarría Castro 
"Diálogos Revista Electrónica de Historia" se publica desde octubre de 1999.

\section{Diálogos está en los siguientes repositorios:}

Dialnet

http://dialnet.unirioja.es/servlet/

revista?tipo_busqueda=CODIGO\&clave_revista $=3325$

\section{Latindex}

http://www.latindex.unam.mx/larga.php?opcion=1\&folio=12995;

\section{UCRindex}

http://www.revistas.ucr.ac.cr

\section{Scielo}

http://www.scielo.cll

\section{eRevistas}

http://www.erevistas.csic.es/

\section{REDALYC}

http://redalyc.uaemex.mx/src/inicio/FrmBusRevs2.jsp?iEdoRev=2\&cvepai=11;

\section{LANIC}

http://lanic.utexas.edu/la/ca/cr/indexesp.html;

Repositorio de Revistas Universidad de Costa Rica

http://www.latindex.ucr.ac.cr/

Directorio y recolector de recursos digitales del

Ministerio de Cultura de España

http://roai.mcu.es/es/inicio/inicio.cmo

DOAJ Directory of open access \& Hybrid journals

http://www.doaj.org/doaj?func=byTitle\&hybrid=1\&query=D

Biblioteca de Georgetown

http://library.georgetown.edu/newjour/d/msg02735.htm

Asociación para el Fomento de los Estudios Históricos en Centroamérica

http://afehc.apinc.org/index.php?action=fi_aff\&id=1774

Universidad de Saskatchewan, Canadá

https://library.usask.ca/ejournals/view/1000000000397982

Monografias

http://www.monografias.com/Links/Historia/more12.shtml

\section{Hispanianova}

http://hispanianova.rediris.es/general/enlaces/hn0708.htm

Universidad del Norte, Colombia

http://www.uninorte.edu.co/publicaciones/memorias/enlaces.htm

Universidad Autónoma de Barcelona

http://seneca.uab.es/historia/hn0708.htm

Repositorio Invenia - Gestión del Conocimiento http://www.invenia.es/oai:dialnet.unirioja.es:ART0000086144

\section{Enlace Académico}

http://www.enlaceacademico.org/biblioteca/

revistas-en-formato-digital-centroamerica/

\section{Electronic Resources}

http://sunzi1.lib.hku.hk/ER/detail/hkul/3987318

Revistas académicas en texto completo http://web.prw.net/ vtorres/

Diálogos se anuncia en las siguientes instituciones y sitios académicos:

Maestroteca

http://www.maestroteca.com/detail/553/dialogos-revista-electronica-de-historia.html

Biblioteca de Georgetown

http://library.georgetown.edu/newjour/d/msg02735.htm

Asociación para el Fomento de los Estudios Históricos en Centroamérica

http://afehc.apinc.org/index.php?action=fi_aff\&id=1774

Universidad de Saskatchewan, Canadá

https://library.usask.ca/ejournals/view/1000000000397982

Monografias

http://www.monografias.com/Links/Historia/more12.shtm

Hispanianova

http://hispanianova.rediris.es/general/enlaces/hn0708.htm

Universidad del Norte, Colombia

http://www.uninorte.edu.co/publicaciones/memorias/enlaces.html

Universidad Autónoma de Barcelona

http://seneca.uab.es/historia/hn0708.htm

Repositorio Invenia - Gestión del Conocimiento

http://www.invenia.es/oai:dialnet.unirioja.es:ART0000086144

Enlace Académico

http://www.enlaceacademico.org/biblioteca/

revistas-en-formato-digital-centroamerical

\section{Electronic Resources}

http://sunzi1.lib.hku.hk/ER/detail/hkul/3987318

Revistas académicas en texto completo http://web.prw.net/ vtorres/

Diálogos Revista de Historia está catalogada por Sherpa Romeo como una revista verde.

La revista electrónica Diálogos es financiada por Vicerrectoría de Investigación de la Universidad de Costa Rica

Citado en: Scielo Dialnet - eRevistas - UCRindex Latindex - REDALYC - DOAJDirectorio y recolector de recursos digitales del Ministerio de Cultura de España

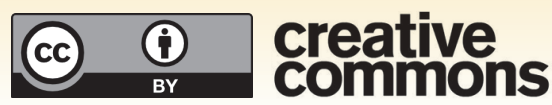




\title{
ACERCAMIENTO CRÍTICO AL HOLISMO EN LA EDUCACIÓN INICIAL: UNA DISCUSIÓN METODOLÓGICA
}

\section{CRITICAL HOLISM APPROACH IN EARLY CHILDHOOD EDUCATION: A METHODICAL DISCUSSION}

\author{
Cynthia Orozco Castro, Maricel Durán Rodríguez \\ Evelyn Fernández Orozco, Sarita Hidalgo Arias \\ Annia Quintanilla Segura, Eva Ramírez Moya
}

\begin{abstract}
Palabras clave
Ecoanálisis, decodificación de lo cotidiano, pedagogía crítica, investigación cualitativa, investigación-acción.
\end{abstract}

\section{Keywords}

Ecoanalysis, decoding day to day, critical pedagogy, qualitative research, action research.

Fecha de recepción: 7 de febrero, 2014 - Fecha de aceptación: 1 de junio, 2014

\begin{abstract}
Resumen
El artículo expone una experiencia de investigación-acción en la cual se llevó a cabo un acercamiento crítico a las actividades y espacios organizados en los distintos ambientes propiciados para la niñez en dos aulas de dos centros infantiles universitarios: Centro Infantil Laboratorio y Centro Infantil Ermelinda Mora. Tomando en cuenta los retos educativos que surgen en nuestra era, la investigación se focalizó en aulas que utilizaban modalidades de atención infantil que pretenden ser holistas y críticas, evaluando la coherencia entre las metas educativas de este paradigma y las acciones cotidianas desde la problematización del currículo oculto y sus implicaciones. Se asume la investigación cualitativa con miras a la decodificación y transformación de los espacios educativos, desde una acción dialógica y propositiva.
\end{abstract}

\begin{abstract}
This lecture results from an action research carried out in two kindergartens: "Centro Infantil Laboratorio" and "Centro Infantil Ermelinda Mora" during this year. These kindergartens base their curriculum on critical and holistic attention to children. Therefore, the objective was to decode and transform educative spaces from a dialogical and transformational perspective. Participants evaluated institutional holistic and critical proposals from the perspective of coherence between educational goals and daily activities, basing problem-solving on the implications of hidden curriculum in both, proposals and processes.
\end{abstract}




\title{
ACERCAMIENTO CRÍTICO AL HOLISMO EN LA EDUCACIÓN INICIAL: UNA DISCUSIÓN METODOLÓGICA
}

\begin{abstract}
Quien no se extraña, quien no se deja atravesar por la pregunta, cautivar por el misterio, no aprende. Quien no se vuelve extranjero de sí mismo, no sabe de sí, no se visita, no se explora, no se aventura, no viaja. Avanzar de esta manera, supone atreverse a pensar la educación como un compromiso.
\end{abstract}

Carmen Emilia García

\section{INTRODUCCIÓN}

Los primeros años de vida son de vital importancia para la formación de nuestra personalidad. En esta edad se construyen las bases de la autoestima; se desarrolla una confianza básica; la seguridad; la autonomía y todos estos aspectos que constituirán la base para el desenvolvimiento futuro como personas pertenecientes a una realidad social.

En nuestro contexto actual el crecimiento de la economía implica una mayor incorporación de las mujeres al mercado laboral. Este hecho hace necesaria una educación inicial de calidad. Pese a esto, aún no existe una orientación más pertinente para las educadoras y educadores en la atención de niños y niñas pequeños.

Como personas ligadas a la educación inicial, hemos sentido la necesidad de buscar propuestas novedosas que ayuden a que se dé un aprendizaje significativo, donde los niños y niñas desarrollen el conocimiento, su personalidad y todo su potencial a través de un ambiente diseñado para abrir posibilidades.

Chaves (2001), en un análisis de la educación preescolar en el contexto nacional, menciona lo siguiente:

Es fundamental estimular la formación de docentes conscientes de la realidad nacional, educadores críticos y creadores de nuevos proyectos educativos a partir de procesos de reflexión y análisis de su práctica pedagógica y del contexto económico, social y político en que se desenvuelven. La creación de currículos holísticos creativos, basados en principios de desarrollo del infante y pertinentes a la realidad socioeconómica y cultural, es un factor que contribuye en gran medida a propiciar aprendizajes significativos y a plantear nuevas necesidades educativas como la ambiental y la ecológica; así como valores de solidaridad y justicia. (Chaves, 2001, p. 12) 
La inquietud de las investigadoras era poder encontrar propuestas innovadoras que ayudaran a transformar la realidad y no solo a analizarla. Por ello se realizó un vínculo con una línea de investigación-acción enfocada en el fortalecimiento de las gestiones curriculares sobre modelos de atención de la niñez centradas en paradigmas holistas y críticos (Chavarría, Orozco, Chacón y Ovares 2000).

Esta propuesta se había venido desarrollando en varios proyectos del Instituto de Investigaciones Psicológicas (IIP) y más recientemente del Instituto de Investigaciones en Educación (INIE), en un modelo de investigación participativa que ha abarcado en distintas fases al Centro Infantil Laboratorio (CIL) y al Centro Infantil Laboratorio Ermelinda Mora (CILEM), (Chavarría, 2003; Chavarría, Obando y Orozco, 2009; Chavarría, Orozco, Chacón y Ovares, 2000; Chavarría, Orozco, Chacón, Ovares y Obando 2000; Orozco, 2005).

Los antecedentes de esta propuesta iniciaron entre 1992-1996, cuando la Dra. María Celina Chavarría desarrolló y puso en práctica la metodología de ecoanálisis en el CIL.

Entre los años de 1997-2002, se desarrolló una proyección de la propuesta de ecoanálisis hacia centros infantiles estatales como el CINAI de Cristo Rey, en un intento por llegar a las poblaciones más necesitadas.

En el año 2005, Orozco realizó un primer acercamiento a la metodología del ecoanálisis en el CILEM de la Sede de Occidente. En los años 2006-2009, tanto el CILEM como el CIL fueron parte de la investigación: Ecoanálisis y decodificación de lo cotidiano en centros infantiles universitarios (Chavarría, Obando y Orozco, 2009).

Tomando en cuenta estos antecedentes, se consideró que el ecoanálisis era una metodología que podía utilizarse como herramienta útil en el proceso de transformar el currículo hacia propuestas más holistas, a la vez que el planteamiento de esta investigación serviría como evaluación formativa a jóvenes docentes de preescolar, algunas de ellas ligadas a estas instituciones.

Las interrogantes planteadas en esta investigación fueron las siguientes:

Desde un paradigma educativo holista y crítico, ¿cómo analizar las actividades y espacios organizados en los distintos ambientes propiciados para la niñez en dos centros infantiles: Centro Infantil Laboratorio (CIL) y Centro Infantil Laboratorio Ermelinda Mora (CILEM), con miras a su decodificación y transformación?

- ¿Qué retos y dilemas educativos surgen en nuestra era que necesitan especial atención y qué preguntas y respuestas dan algunos teóricos holistas sobre el tema? 
- ¿Cómo tomar conciencia de las implicaciones éticas, filosóficas y axiológicas de la visión de mundo y de las prácticas educativas?

- ¿Cómo contribuir a la transformación que como seres humanos hemos de experimentar para ser agentes transformadores de la educación desde la acción- reflexión y la reflexión-acción como insta Paulo Freire?

- ¿Cómo decodificar el currículo oculto y sus implicaciones en aulas que utilizan modalidades de atención infantil que pretenden ser holistas y críticas?

- ¿Cómo crear una comunidad de aprendizaje, interrogándonos sobre la comunicación amorosa de esos seres que se inician en la vida?

- ¿Cómo fomentar el sentido de indagación y creatividad de las docentes como transformadoras?

Para efectos de este artículo, se hará énfasis en los aspectos metodológicos de esta investigación, para resaltar el aporte del ecoanálisis dentro de la investigación acción.

El marco teórico se apoya en la pedagogía crítica de Paulo Freire (1970), en una visión de la educación liberadora y transformadora, la actitud dialógica y la necesidad de formar docentes conscientes de la realidad, críticos y reflexivos. Se basa también en los preceptos de la educación holista (para una mayor profundización de estos aspectos ver Chavarría, Obando y Orozco, 2009; Durán, Fernández, Hidalgo, Quintanilla y Ramírez, 2010).

\section{METODOLOGÍA}

La propuesta se realizó con un enfoque cualitativo, desde la modalidad de investigación acción crítica, cuyo objetivo se encuentra dirigido a desentrañar los significados de las acciones humanas en un contexto histórico específico.

Se analizaron las actividades y espacios organizados en los distintos ambientes propiciados para la niñez, en este caso en dos centros infantiles de la Universidad de Costa Rica que han implementado acciones con miras a acercarse al paradigma holista: el CIL y el CILEM. Se hizo referencia a las relaciones e interacción que se dieron entre los niños y niñas hacia sus iguales, las personas adultas y el ambiente en el cual se desenvolvían. Todo con el fin de valorar una metodología de marco 
abierto y aportar propuestas que contribuyeran con el mejoramiento y la continua transformación de los espacios educativos.

Tal y como señala Sandín (2003), este enfoque "Contribuye a la reflexión sistemática sobre la práctica social y educativa con vistas a la mejora y al cambio tanto personal como social" (p. 164). Por lo tanto, desde la investigación acción, esta práctica estuvo dirigida a la comprensión e interpretación de un contexto educativo, con miras a desarrollar algunas transformaciones acordes con los requerimientos del holismo como paradigma educativo.

\section{TÉCNICAS DE INVESTIGACIÓN}

Se realizaron observaciones participantes en las cuales se registraron las apreciaciones del ambiente y las relaciones que en él se desarrollaban en un aula de cada centro infantil. Para las observaciones se utilizaron diferentes guías de observación que orientaron la mirada de las investigadoras:

- Interrogantes Claves para el Ecoanálisis (Chavarría, 2006)

- Problematización en torno a una educación inicial holista y crítica (Chavarría, Orozco, Ovares, Obando y Rivera, 2007)

Un punto importante es que los valores desde los cuales se realizó la investigación fueron explicitados, visibilizados y no asumidos como neutrales. Los valores se reconocieron desde el paradigma elegido, el contexto donde se desarrolló la práctica y las teorías que la fundamentan.

Parte de las premisas ontológicas acordes al paradigma holista de la educación asumen que el ser humano:

Es un ser dotado de inteligencias múltiples en su individualidad biológica, con diferentes perfiles cognoscitivos y estilos de aprendizajes y, consecuentemente, con diferentes habilidades para resolver problemas. Un individuo que aprende, representa y utiliza el conocimiento de modo diferente, que conoce el mundo de una manera específica dependiendo del perfil de las inteligencias que posee, del contexto, y de la cultura en que fue generado. Un aprendiz que es producto y síntesis de experiencias personales interiores y exteriores, un sujeto que es histórico y, al mismo tiempo, sujeto de la historia, constructor de su propia historia. Un ser espiritual en busca de su propia trascendencia, en un viaje individual y colectivo en busca del significado de la vida. (Moraes, 1999, p. 43) 
Visto el ser humano como un ser integral, surge la necesidad de ofrecerle la oportunidad de potenciar todas sus habilidades, de responder a sus intereses y necesidades para llevarlo a desenvolverse y desarrollarse plenamente. Esto implica una indagación crítica de la realidad social. Nace la necesidad de decodificar lo cotidiano y poner en evidencia el currículo oculto para esclarecer la realidad educativa en la actualidad.

En cuanto a los sujetos de la investigación, estos fueron conformados por las docentes y asistentes de los grupos escogidos y sus estudiantes. En el caso del CILEM, 4 niños y 4 niñas de edades entre los 8 meses a 2 años y medio, y en el CIL 18 niños y niñas de edades entre los 4 años y 3 meses y los 5 años y 2 meses.

La primera etapa de esta investigación comprendió la lectura, discusión grupal y problematización teórica.

Luego de cada una las observaciones realizadas y durante las reuniones grupales, se sistematizó la información por medio de triangulaciones. Estas consistieron en elaborar una puesta en común a partir de las percepciones que tuvo cada una de las investigadoras, por medio de una relación de análisis entre el sustento teórico, las prácticas y ambientes observados y las interpretaciones de estas. Este espacio de discusión y problematización sobre las teorías y sus implicaciones en las prácticas educativas, permitía ir afianzando la reflexión sobre la acción y analizar la congruencia de las acciones educativas a la luz de las premisas del holismo. Luego la discusión giraba en torno a la visualización de cómo mejorar los procesos.

Se seguían los pasos del ecoanálisis, o análisis de los espacios y situaciones que conforman el acontecer cotidiano, como una herramienta para decodificar lo cotidiano. De acuerdo con Chavarría y Orozco (2006), ello implica mirar y analizar, con ojo crítico, el diálogo yo-mundo que se da en las rutinas diarias de un centro infantil, pues de esa forma se descubren valores subyacentes a las metas de desarrollo. El análisis ecológico intenta hacer manifiestos los significados explícitos de los acontecimientos, como medio para facilitar cambios en las relaciones entre las personas y sus mundos.

Las devoluciones se realizaron de la siguiente manera:

En el Centro Infantil Laboratorio Ermelinda Mora, después de realizar la primera ronda de observaciones, tres de las investigadoras triangularon y analizaron la información para presentarla y discutirla con la docente encargada del grupo del nivel I del CILEM. La segunda devolución se hizo en presencia de tres de las investigadoras y la docente encargada del grupo. En esta ocasión se hicieron evidentes los cambios propuestos en la primera puesta en común.

En la tercera devolución participaron las tres investigadoras y la docente del primer nivel. 
Las devoluciones consistieron en la lectura del documento y en el diálogo de las fortalezas, aspectos por mejorar y retos que se presentaron de acuerdo con las observaciones realizadas. Durante las conversaciones, tanto la docente encargada como las investigadoras estuvieron anuentes a las recomendaciones, retos y aspectos por mejorar.

El diálogo se dio en un ambiente de respeto, en donde se analizaron cada uno de los aspectos y se escucharon los diferentes puntos de vista para lograr una mayor coherencia con los principios de la educación holista. El objetivo de dicho proceso fue enfatizar la búsqueda de soluciones creativas para transformar el ambiente situación.

En el Centro Infantil Laboratorio, la primera devolución realizada a la docente encargada del grupo se dio por parte de dos de las investigadoras y se contó con la participación de una observadora que realizaba una investigación en el lugar.

Durante la puesta en común se vivió un ambiente muy respetuoso y cordial en el que se destacaron en su mayoría aspectos positivos y algunos que se consideraron necesarios de problematizar y transformar para una mejor coherencia de la filosofía. Ante estos cambios que sugirieron, la docente mostró en todo momento una actitud muy positiva y abierta que hizo fácil el diálogo con ella.

Para la realización de la segunda devolución en el CIL, correspondiente a la tercera observación, se contó con la presencia de dos de las observadoras y la participación de varias personas que laboran en la institución, entre ellas, la docente del nivel IV, la asistente de este grupo, la directora del CIL y la coordinadora pedagógica, pues todas fueron invitadas por la docente y deseaban participar del proceso. Se plantearon aspectos positivos y otros que representaban un reto y era necesario corregirlos, todos fueron comentados de manera muy respetuosa por las investigadoras y por las personas del CIL, se sintió una muy buena vinculación de grupo con la investigación, además de una interacción más rica en cuanto a visualización de proceso. Al igual que la ocasión anterior la docente aceptó sin problema los cambios sugeridos y en general la reunión transcurrió en un ambiente dialógico, asertivo y contó con los aportes de todas las presentes.

Este proceso se realizó en tres ocasiones, como cierre de cada proceso se realizaron las triangulaciones.

De este diálogo o problematización conjunta, se derivaron los significados, interpretaciones y visiones de lo observado. Se esclarecieron en equipo los múltiples y posibles significados de lo vivido, con el fin de llegar a acuerdos para comenzar a focalizar la atención en espacios-situaciones particulares, en aras de las transformaciones iniciales posibles.

Posteriormente se llevó a cabo otro ciclo de observaciones, ahora intentando visualizar aquellos aspectos que podrían representar mejoras en términos de necesidades detectadas. 


\section{PASOS DEL ECOANÁLISIS}

A continuación se detallan los pasos del ecoanálisis, de acuerdo con Chavarría y Orozco (2006):

Planteando las primeras interrogantes sobre los ambientes existenciales y el currículo oculto

Para hacer un ecoanálisis en un contexto educativo, siguiendo a Chavarría y Orozco (2006), un primer paso insta a mirar las actividades llevadas a cabo como el conjunto de ambientes-situaciones en la cotidianidad del centro infantil. Se intenta una aproximación a los conceptos, rituales, tiempos y estructuras del modelo educativo para intentar comprender cómo se escenifica el mundo de la educación y cómo podrán estar experimentándolo las personas involucradas.

Las observaciones y anotaciones permiten interpretar cómo están vivenciando los niños y niñas el mundo en cada ambiente-situación del aula. Asimismo, cómo parece vivenciarlo la docente y cuál es la concepción de niñez y de aprendizaje que se puede deducir de sus acciones y gestos, aunque al preguntársele la docente asuma otro tipo de valores o principios. En general, se problematiza sobre estas vivencias.

En esta etapa una interrogante clave es: ¿Coexisten versiones filosóficas que luego son contradichas por las acciones, gestos y prácticas curriculares?

Según lo registrado en las observaciones realizadas y tomando en cuenta los instrumentos de observación del ecoanálisis, se evidenciaron categorías de análisis tales como: Ambiente existencial, Comunidad de aprendizaje, Solidaridad y equidad entre hombres y mujeres: Igualdad de oportunidades y conciencia de género, Apreciación y conocimiento activo de la naturaleza: Visión de sostenibilidad, Sentido cultural del tiempo y el espacio.

Para ejemplificar, a continuación se menciona una de las observaciones referentes a esta primera etapa, en la que además, los niños y niñas estaban en proceso de adaptación al centro infantil.

En las observaciones realizadas se evidenció que durante todo el tiempo se les validaban a los niños y las niñas pequeños sus emociones y sentimientos.

En el CILEM:

\footnotetext{
Un niño pequeño, llega de su casa llorando, la docente le brinda contención, lo lleva a un banco y cariñosamente lo sienta en sus regazos y le pide a una de las niñas que le dé un abrazo a su compañero. La niña se acerca con ternura y lo abraza. El niño se tranquiliza y ella le invita a jugar con unas tortugas plásticas que se encuentran en un recipiente con agua y jabón.
} 
Se evidencian sentimientos, como el derecho a llorar, la ternura, el cariño entre las y los miembros del grupo, y la manera en la que la docente resuelve la situación, pues el niño no deja de llorar por resignación ni cansancio sino porque se le da contención, se le hace saber que en ese lugar hay personas que respetan sus sentimientos y que le quieren. El infante se tranquiliza al sentirse seguro y querido y se dirige a jugar. (E. Ramírez, observación de clase, 27 de abril, 2009)

En las edades de 8 meses a 1 año y medio, el acompañamiento y la contención de las docentes hacia los chicos y chicas son necesarios. Es por esta razón que pudo verse constantemente algún chico o chica en brazos. Estos procesos se acompañaban de besos y abrazos por parte de la maestra, lo que creaba fuertes vínculos y evidenciaba cómo se atienden las necesidades de cada persona.

Este otro ejemplo, en el CIL:

A eso de las ocho y cuarenta se avisa que la actividad de siembra de árboles va a dar inicio y pasan al comedor para dar indicaciones. Cada niño y niña va acompañado y acompañada por una o un familiar, se pasa a recoger los árboles al aula verde y se camina cerca del planetario. Funcionarias de la Universidad de Costa Rica, del Departamento de Gestión Ambiental de la Universidad dieron una pequeña charla acerca de la importancia de sembrar árboles. Explicaron el procedimiento para realizarlo de forma muy sencilla y con vocabulario muy entendible, aunque se dirigían principalmente a los y las adultas presentes, para que ellos y ellas luego se lo explicaran a sus hijos, hijas, nietos o nietas. Luego, cada quien se dispuso a buscar un hueco para plantar su árbol. Las personas, las y los infantes estaban muy motivados y motivadas.

Las indicaciones se dan claramente y al salir detrás de muchas de las personas, se pudo observar una gran cantidad de gente movida por la esperanza de un mañana mejor, por el deseo de preservar, contribuir con la naturaleza y sensibilizar a los y las pequeñas, lo cual me llena de alegría. Se sentía un ambiente de cooperación y satisfacción personal, las personas caminaban y conversaban entre sí con una sonrisa en el rostro. (E. Ramírez, observación de clase, 5 de junio, 2009)

Al mismo tiempo que se visibilizaban los aspectos positivos de este primer acercamiento, se planteaban las primeras interrogantes sobre el currículo oculto. Por ejemplo:

Una niña pequeña trabaja en trasvasar las conchas y lo hace muy bien, pero lo hace en el estante, no traslada el material a la mesa porque esta le queda 
muy lejos y el material es pesado... Es necesario reubicar las mesas del centro del aula para que queden más cerca de las actividades que requieren de una, porque la niña que no trasladó la bandeja con las conchas tal vez prefirió no hacerlo por lo lejos que le queda la mesa y por el peso de la bandeja. (E. Fernández, observación de clase, 12 de mayo, 2009)

\section{Problematización y escogencia de ambientes existenciales específicos, así como de niños y niñas a quienes dar seguimiento, con miras a focalizar lo posible}

El objetivo de este momento del ecoanálisis es que el equipo problematice sobre los significados de las acciones observadas, así como su congruencia con una cosmovisión holista. Para ello, tal y como se planteó anteriormente, se discutieron las observaciones, triangulando la información para elegir algunos aspectos y analizarlos a la luz de lo esperable en una propuesta holista. En este apartado se mencionan las interrogantes que se consideran claves en esta problematización. En el paso 3 del ecoanálisis se plantearán las propuestas que el equipo le hizo a cada docente para mejorar.

Para ejemplificar, un aspecto que se cuestionó estuvo relacionado con el tipo de videojuegos utilizado en la computadora por parte de los niños y niñas. Estos fueron considerados violentos y no contribuían a la formación de seres humanos pacíficos y solidarios, ni a un ambiente que promueva la cultura de paz.

Los niños y las niñas están en la computadora viendo un juego de Hot Wheels. Para algunos es incómodo, ya que no ven bien. Las imágenes de accidentes de autos se acompañan de gritos, sobre todo de los niños. Junto a la computadora hay una pizarra donde se colocan los nombres de quienes les corresponde el uso de la computadora ese día. Estos no han llegado por lo que la maestra cedió el uso a otros bajo el entendido de que cuando lleguen deben darles la computadora. La asistente pega los nombres con cinta adhesiva.

El uso de la tecnología como herramienta se puede convertir en una forma divertida de aprender, no solo porque se encuentra presente en la cotidianidad global, sino porque bien utilizada puede ayudar a diversas habilidades. En la observación se sintió que el juego puesto a disposición de los niños y niñas genera disturbio en el aula. Los niños alimentan su estereotipo de "macho" de pelea, competencia, velocidad y otras que alimentan la diferencia de género, ya que las niñas están como espectadoras pero no se involucran directamente.Se considera importante que la escogencia del material sea mejor cuestionada. Podría ser importante tratar de hacer parejas niño y niña, ya que según la lista que vi en pocas ocasiones coincide esto, con el fin 
de promover las relaciones entre géneros. (S. Hidalgo, observación de clase,

29 de mayo, 2009)

Es importante considerar que los videojuegos son herramientas de aprendizaje muy poderosas. En este caso el aprendizaje orientado hacia la violencia contradice los valores que se quieren fomentar, basados en la resolución creativa de conflictos y una educación para la paz.

El acceso que los niños y niñas tengan de la tecnología es muy importante siempre y cuando los adultos a cargo elijan lo que ponen a su disposición, por esta razón, los retos visualizados fueron los siguientes:

- ¿Cómo ofrecer herramientas tecnológicas que permitan un aprendizaje significativo y gozoso para los niños y niñas, congruentes con una visión holista?

- ¿Cómo establecer límites de respeto en la utilización de ciertos espacios sin alterar la libertad de escogencia?

En cada categoría de análisis se trabajó con preguntas orientadoras, tales como las anteriores.

\section{De lo inconsciente a lo consciente: Visualización de mundos posibles}

Después de realizar las primeras 10 observaciones, el equipo de trabajo analizó una serie de aspectos. Se partió de las discusiones que se generaron con el fin de visualizar alternativas.

En esta fase de triangulación se señalaron fortalezas, aspectos por mejorar y retos en cada una de las aulas observadas. Se inició con las fortalezas y posteriormente se analizaron los retos y las propuestas visualizadas, siguiendo valores de una visión holista.

En este momento del proceso, se intentó poner palabras en las sensaciones o percepciones que un determinado ambiente-situación suscitaba, preguntándose, por ejemplo: ¿Qué posibilidades sugiere este ambiente-situación? ¿Qué posibles acciones se necesitarían para mejorar?

Como comunidad de aprendices, se necesita de un proceso de reflexiónvisualización en torno a los valores que se quieren ver plasmados, poniendo el énfasis en el lenguaje de lo posible. Cada partícipe intentaba visualizar transformaciones posibles, en torno a valores particulares. Por ejemplo: ¿Cómo darle más calidez y oportunidades significativas a este espacio? (Chavarría y Orozco, 2006). Estas sugerencias fueron discutidas, comentadas y analizadas con las maestras de forma dialógica. 
Por ejemplo, en el CILEM, a partir de la decodificación de lo cotidiano se pudo observar una gama de aspectos positivos que favorecían la armonía, calidez y desarrollo de este grupo de niños y niñas que compartían el aula del nivel I. Dichos aspectos daban validez a la funcionalidad del ecoanálisis como metodología de trabajo.

En este contexto sobresalía un ambiente físico confortable, se evidenciaba la presencia de materiales y diferentes actividades cotidianas que incitan a la libertad y la curiosidad. Dichos materiales a disposición de los niños y niñas brindaban oportunidades para la vida diaria que promovían la equidad de género. Al mismo tiempo representan tareas y actividades para el aprendizaje. Se evidenció el contacto real de los niños y niñas con la naturaleza, lo que generó actividades significativas en su apreciación y valoración.

Estos ambientes preparados para que el niño y la niña desarrollasen sus habilidades por medio de trabajos de la cotidianidad, adquieren un gran valor para ellos y ellas cuando se analiza que estos no representan simples juegos, sino retos y aprendizajes significativos. "Esto implica, por ejemplo, concordar con su necesidad de construirse a sí mismos como seres útiles, de conocer el mundo e interactuar con la comunidad, en formas más productivas y profundas de lo que usualmente pensaríamos como juego" (Chavarría, Orozco, Ovares, Obando y Rivera, 2007, p. 59).

Por otra parte, se hizo visible la construcción de una comunidad de aprendices donde cada persona es importante y respetada, además cumple con sus responsabilidades con el grupo. Se evidenciaron actitudes de solidaridad por parte de los niños, las niñas y la docente.

En la mayor parte de las actividades se observó cómo los niños y las niñas tenían la libertad de elegirlas y las realizaban por su propio interés durante largos periodos, lo cual favorece el proceso de autonomía.

Sin embargo, se percibieron rutinas tradicionales como conversaciones prolongadas en círculo y monótonas actividades de arte para todo el grupo, en las que la mayoría no estaba interesada. Por ende, una recomendación se dirigió a evitar que se cortaran espacios de interés para cumplir aspectos en los que los y las pequeños no tenían ningún interés.

Desde una perspectiva filosófica holista, nuestra práctica educativa implica respetar la autogestión. Cada uno de nuestros infantes posee diferentes intereses, experiencias previas, contextos, motivaciones, entre otros. Por lo tanto, estos son la brújula que indica cómo debe funcionar el sistema educativo para responder a sus necesidades.

Entendemos así que los procesos de enseñanza y aprendizaje producidos en las escuelas infantiles no son aislados e individuales, y que no se desarrollan con independencia del contexto, sino que, por el contrario, son momentos de 
construcción de conocimiento que se producen en la relación con los otros (adultos y niños). (Osoro y Meng, 2008, p. 20)

Se hizo necesario comentar algunos aspectos que resultaban incongruentes con la metodología utilizada, los cuales era esencial visibilizar.

Al respecto, se hallaron principalmente algunos elementos que restaban armonía, estética y organización al ambiente preparado. Por ejemplo, a simple vista no quedaba clara la distribución por espacios: las mesas de trabajo se encontraban lejos de estos y no estaban a la altura de los niños y las niñas, existían pocos elementos interculturales y que favorecieran el desarrollo de los sentidos. Otro aspecto que se destacó se refería al papel de las docentes en la cotidianeidad, pues se pudo visualizar la necesidad de una mejor comunicación.

Ante los aspectos citados se realizaron algunas propuestas derivadas del ecoanálisis para mejorar las condiciones del aula de trabajo. A grandes rasgos mencionan las siguientes:

- Brindar mayor organización dentro del aula. Esto suponía que las áreas de trabajo estuvieran claramente delimitadas tomando en cuenta la temática y reforzándolas con fotografías de los niños y las niñas trabajando.

- Enriquecer diferentes áreas como la de vida cotidiana.

- Incorporar material sensorial y de contacto con la naturaleza y las culturas.

- Cambiar los materiales que ya no producen interés.

- Incorporar un proyecto para un manejo adecuado de los desechos.

Para llevar a cabo dichas propuestas era necesaria una decodificación reflexiva de lo cotidiano. Esto quiere decir "crear y recrear el espacio, pensar y repensar los materiales que utilizamos, gestionar los tiempos, tanto individuales como colectivos..." (Osoro y Meng, 2008, p. 24).

En el CIL se observaron muchos aspectos positivos. Entre ellos: la distribución adecuada del aula, las áreas con ejercicios y juegos que resultaban significativos para los niños y niñas. Igualmente, promovían en ellos y ellas un reto cognitivo y un mayor aprecio por la cultura y el amor por la naturaleza.

Además, fue notorio que las docentes trataban de cultivar un ambiente de paz, en el que cada persona dentro de él se sintiera especial y segura. Se percibió también que de parte de las adultas existe respeto hacia los y las pequeños, hacia lo que tienen que decir, sus opiniones y decisiones.

Pero además, era necesario problematizar sobre algunos aspectos que resultaban incongruentes con la metodología utilizada y con la filosofía por seguir, el holismo. Estos aspectos probablemente pasaban desapercibidos por las propias docentes, formando de ese modo un currículo oculto que era necesario visibilizar. $\mathrm{Al}$ respecto, se hallaron principalmente algunos elementos que restaban armonía, 
estética y organización al ambiente preparado, como objetos en mal estado, algunos que se observaban desorganizados y fuera de su lugar, otros que ya no parecían ser utilizados por los niños y niñas.

El otro aspecto que se problematizó se refería al papel de las docentes en la cotidianeidad. En este caso lo más notorio fue que había que mejorar la comunicación entre docente y asistente.

Para tratar de ofrecer una solución a los aspectos problematizados se realizaron algunas propuestas de materiales que podrían colocarse en ciertos espacios, de manera que muebles y objetos no hicieran perder la estética del aula, de modo que estos se mostrasen más organizados, lo cual era sumamente necesario.

\section{Puesta-en-común en torno a mundos posibles}

Luego de las observaciones realizadas en los Centros Educativos elegidos, y específicamente en los niveles que respondían a una filosofía holista, se realizó una puesta en común con las docentes encargadas de dichos grupos.

Después de visualizar dimensiones de lo posible, el equipo desarrolló discusiones detalladas e intensivas, acerca de las experiencias y sentimientos que los ambientes-situaciones elegidos generaban, sobre los esbozos de lo posible. Intentó acercarse en forma empática a las situaciones contactadas y exploradas (Chavarría y Orozco, 2006). De esta forma se conjuga la crítica social con la visualización de lo posible (Rodríguez, 2007).

Se registró cada sesión para realizar un análisis de las dimensiones o conceptos claves.

En el CILEM, al decodificar la experiencia educativa ofrecida a los niños y niñas, el equipo pudo observar que era necesario propiciarles una serie de elementos y situaciones que permitieran el desarrollo de las actividades y experiencias de una forma fluida y provechosa. Posterior a esta decodificación, en equipo se formularon opciones viables que colaboraran al mejoramiento del ambiente existencial de los niños y niñas.

Luego se realizó una puesta en común, por parte de las integrantes del grupo de investigación y la docente a cargo de los niños y las niñas, donde se expusieron los puntos en discusión, siempre con una actitud de diálogo y respeto.

La discusión se realizó en el CILEM con la docente encargada del grupo y dos investigadoras. Esta se llevó a cabo mediante la exposición de las fortalezas, aspectos por mejorar y los retos que se hicieron evidentes.

Se comentaron dichos aspectos y la docente encargada iba aportando sugerencias y explicando el porqué de los aspectos observados, siempre en términos de diálogo.

Las fortalezas eran realmente significativas. Evidenciaban el progreso de 
romper con los esquemas tradicionalistas y emprender la tarea hacia una visión más holista, teniendo como gran reto trabajar con niños y niñas de tan corta edad. La discusión generó cambios en toda el aula.

Posteriormente se realizó otra ronda de observaciones donde se destacaron los cambios realizados por las docentes partiendo de las sugerencias discutidas. Esto demostró el interés y compromiso hacia la comunidad de aprendices. Se propiciaba un ambiente más familiar y de pertenencia en el aula, murales de fotografías de los niños y niñas trabajando, materiales y mobiliario más accesibles, organización del espacio e incorporación de materiales nuevos y diversos, sobre todo de la cultura costarricense, elementos como vasijas, carretas, bolsa de chorrear café, por mencionar algunos. Otros aspectos importantes son el acompañamiento, por parte de la docente y la asistente, de los niños y niñas, la flexibilidad de las situaciones de aprendizaje y en general la construcción de un ambiente preparado para dar lugar a un aprendizaje en lo cotidiano basado en un modelo holista. Este ejemplo evidencia lo anterior:

En esta observación, el aula luce muy diferente a la primera vez. Se nota que se tomaron en cuenta las recomendaciones que se dieron en la triangulación. Es muy importante resaltar que se hayan tomado en cuenta las sugerencias dadas en la triangulación para hacer modificaciones dentro del aula y que ofrecieran un ambiente más preparado para los niños.

El poder establecer diálogos y reflexionar sobre las acciones que se están llevando a cabo dentro de la comunidad educativa es sumamente importante porque así se dan los cambios necesarios para ir haciendo del proceso algo aún más significativo. (E. Fernández, observación de clase, 18 de junio, 2009)

Otro ejemplo en el CIL:

Se observa al niño que en la observación anterior presentaba serios problemas en su relación con los y las demás. Se acerca al espacio de ciencias después de haber merendado tranquilo y le pregunta a la docente si puede jugar con un material. Ella con tono alegre y amigable le dice que sí claro. Él toma una caja con un material de piezas plásticas de ensartar (tipo legos), el cual tiene unos tubos que se encogen y estiran como resorte. Es un material nuevo, muy atractivo y al niño parece llamarle mucho la atención. Le dice a un compañero que se acercó interesado en lo que él hacía, que está armando una araña. El compañero accidentalmente le bota una pieza, el niño le dice que no pero con tono natural, sigue armando la araña. Le ha puesto unas patas con los 
tubos que son como resorte, ahora hace caminar la araña, lleva cerca de 30 minutos en ese juego y está sumamente concentrado.

Este niño ha mejorado mucho su forma de estar en el mundo en poco tiempo. La última vez que se observó no duraba ni 5 minutos con un material, no lograba concentrarse, estaba constantemente buscando problemas con los y las demás. Es maravillosa la sensación al verlo. Está profundamente concentrado, le encanta la araña que hizo, se nota orgulloso de su trabajo, nada que pase a su alrededor logra distraerlo, en este momento está experimentando un gran gozo, está contento con lo que hace, lo vi sonreír y eso conmueve porque la ocasión anterior que se le observó no se le vio gestos de alegría en él. (M. Durán, observación de clase, 31 de julio, 2009)

Otro ejemplo:

\begin{abstract}
Ahora juegan en los espacios del aula. En la computadora la docente ha puesto un video muy interesante sobre la época de los cavernícolas, la invención del fuego y su forma de vida. Hay muchos niños y niñas que lo quieren ver, cada vez se acercan más, M... les va explicando lo que va ocurriendo en el video, existe un gran interés y concentración.

Es excelente que se pueda aprovechar tan bien un recurso como la computadora, es muy adecuado que desde su edad los niños y niñas tengan acceso a la tecnología, experimenten sus utilidades y les facilite la adquisición de conocimientos interesantes. (M. Durán, observación de clase, 31 de julio, 2009)
\end{abstract}

Esta opción ofrecida, contrasta con el video de la primera observación.

\title{
Actualización de lo posible y reiteración del proceso
}

Los próximos pasos del ecoanálisis comprenden el reinicio de los procesos de observación, visualización de posibilidades, puesta en común, con el fin de generar nuevas transformaciones. Y así sucesivamente. Tanto las reacciones de chicos y chicas como las del equipo darán las pistas necesarias.

Los procesos humanos son inacabados y mejorables, por esta razón es de suma importancia no ser determinante o generalizar en las situaciones que se presentan día a día en una jornada de trabajo en preescolar, así como en las situaciones propias de la vida de cada individuo. De ahí la importancia de seguir observando los procesos y de sacar el mayor provecho de aquello de lo que se pueda aprender para mejorar el ambiente, el diálogo y las propuestas por desarrollar. 
En el transcurso del proceso, se observó cómo el ambiente del aula cambiaba tanto físicamente como en las relaciones que se establecían dentro de la comunidad de aprendizaje.

\section{CONCLUSIONES}

Al concluir esta investigación se ha valorado la importancia de la pedagogía crítica como herramienta de análisis para responder al cambio de época y las necesidades humanas que de esta devienen: un análisis crítico de la realidad.

Los retos actuales para el mejoramiento y la transformación de la educación inicial son múltiples y complejos. Siendo la propuesta nueva y amplia de un currículo holista, se considera que este trabajo es un acercamiento inacabado, con miras a mejorar el contexto en el que se trabajó.

Fue de gran ayuda trabajar con preguntas orientadoras que permitieron plantear metas claras y ver los retos que se tenían por delante. Sin embargo, no se considera que las respuestas sean lineales ni cerradas, sino que muchas de estas interrogantes orientaron nuevos planteamientos, que se convierten en un insumo para la reflexión-acción.

La propuesta de una educación holista está en construcción y se considera importante estudiar más sobre el tema, pues los principios de esta propuesta son más acordes con las necesidades del momento histórico actual. Sin embargo, dada la complejidad, y la ruptura epistemológica, axiológica y ética, implica analizarla más profunda y críticamente, lo cual trasciende los alcances de esta investigación.

Las investigadoras apreciaron la importancia de establecer currículos de marco abierto que posibiliten un ambiente preparado, encaminado a una educación para un desarrollo sostenible, que se oriente a tomar conciencia de que somos seres interdependientes y capaces de transformar la realidad. Ante este reto, es imprescindible un currículo que evalúe constantemente su actuar y su compromiso con la formación de personas espiritualmente más humanas e integrales, lo cual a su vez tiene implicaciones éticas, epistemológicas y axiológicas.

El proceso de decodificación que se realizó luego de cada ciclo de observaciones, permitió que las docentes ampliaran su visión de la realidad de la propuesta educativa que ofrecían a los niños y niñas. Esto las llevó a valorar las proposiciones sugeridas por el grupo investigador a través del diálogo y de este modo realizar los cambios que se consideraron pertinentes para la transformación con miras a nuevos mundos posibles.

Decodificar lo cotidiano dio la posibilidad de revelar con ojo crítico lo que estaba más allá de lo que se podía percibir a simple vista. Extraer aspectos de un 
currículo oculto presente en los ambientes observados y que pasaba desapercibido por falta de un análisis profundo de la cotidianeidad, para realizar cambios que permitieran solventar los retos encontrados, en congruencia con un modelo más holista. Además, permitió tomar conciencia de lo que cuesta decodificar el currículo oculto, pues al ser naturalizado, se ve lo que pasa en el aula como algo "normal". La mirada interdisciplinaria colabora en visibilizar estos aspectos.

Dentro de la educación inicial existen diversas problemáticas que dificultan la calidad y el valor de esta. Por ejemplo, la falta de recursos económicos para ofrecer ambientes idóneos para la atención. En este caso, en ambas instituciones observadas se hizo clara la necesidad de modificar y reemplazar recursos materiales. Lo cual se hace más significativo en centros infantiles pertenecientes a la Universidad de Costa Rica, que deben actuar como centros de proyección a la comunidad.

Dichas dificultades, por mantener la congruencia con modelos de marco abierto tendientes al holismo, hicieron que se rescatara la importancia del compromiso que muestran las docentes día a día para ofrecer a los niños y las niñas el ambiente existencial más idóneo, agradable y cálido. El mérito que reciben estas educadoras por su desempeño se refleja en la respuesta de los niños y niñas ante el ambiente situación que se les ofrece. Se evidencia en el disfrute expresado por ellos y ellas al interactuar en los diferentes espacios y establecer relaciones interpersonales de un modo pacífico, libre y flexible.

El proceso de decodificación y diálogo permitió conocer los puntos de vista de cada uno en los diversos procesos de la investigación, y triangular dicha información para analizar los criterios con miras a proponer transformaciones positivas y cercanas a la realidad. El diálogo y los cuestionamientos realizados en conjunto promovieron el idear soluciones más creativas y completas para solventar los retos que surgían continuamente. Este hecho tiene una implicación ética, filosófica y axiológica, de la visión de mundo y de las prácticas educativas, basada en el diálogo, el respeto y el trabajo conjunto.

El hecho de participar observando, decodificando, proponiendo y dialogando sobre una metodología novedosa en la educación costarricense es muy enriquecedor pues, tanto a nivel personal como profesional, se valora la necesidad de transformar la visión del sistema educativo tradicional que impera en este momento. Este se caracteriza por ser rígido, con tiempos limitados para cada actividad, rutinario, con relaciones de verticalidad entre docente y estudiante, entre otros. Mientras que el basado en una visión holista posee ambientes de libertad, sentido del tiempo flexible, relaciones horizontales, donde los espacios que se ofrecen están pensados para favorecer el interés de quienes interactúan con ellos, respondiendo a sus necesidades. 
Al utilizar una metodología dialógica se puede tener acceso a las ideas, opiniones y sugerencias de las diversas personas involucradas en el proceso y así enriquecer el conocimiento sobre la metodología y filosofía empleadas en esta investigación. Además, el diálogo nos permitió ir transformando la realidad del aula y el contexto en el que está inmersa.

De esta forma, se contribuye al cambio que como seres humanos se ha de experimentar para ser agentes transformadores de la educación desde la acciónreflexión y la reflexión-acción como insta Paulo Freire.

Recapitulando, en las observaciones relevantes pudimos evidenciar que en edades tempranas los niños y las niñas van construyendo las diferencias entre los hombres y las mujeres, incorporando roles culturales guiados por el modelaje de la sociedad en la que están inmersos. Por lo anterior, se concluyó que la creación de un ambiente pensado críticamente abre oportunidades para que ellos y ellas participen en tareas que van en contra de los estereotipos de género estipulados; por ejemplo, jugar legos y carpintería, bañar un muñeco, barrer, entre otros. Al proporcionarles dichas oportunidades de desenvolverse, los niños y las niñas actúan de formas no tradicionales y se rompe con estos esquemas.

Se evidenció cómo el tener una relación respetuosa entre los niños y niñas y las docentes, no significa que se pierda el respeto y los límites dentro de la jornada. Por el contrario, esto abre posibilidades para una comunicación más fluida y de confianza. Además, un tiempo sin las consignas que tradicionalmente se conocen (como la canción que se canta antes de ir a lavarse los dientes, que siempre es la misma) es más flexible. Una mayor libertad lleva a los pequeños y pequeñas a realizar los trabajos en los espacios que son de su agrado y durante el tiempo que consideren necesario. Esto hace que se valoren los ritmos significativos del aprendizaje.

Por otro lado, el papel que juega la docente ya no se limita a ofrecer una simple clase magistral, sino que trasciende lo que tradicionalmente se conoce, ubicándose en una posición de diseñadora y facilitadora de oportunidades para aprendizajes significativos. Esto lleva a replantar las implicaciones éticas, filosóficas y axiológicas de las prácticas educativas y a buscar nuevas alternativas que permitan llevar a que la educación inicial esté más acorde con los retos y la realidad del mundo de hoy.

En las observaciones realizadas, se pudo percibir que por medio del ambiente que está previamente preparado para el aprendizaje en lo cotidiano se adquieren valores referentes a la responsabilidad, la cooperación con tareas cotidianas, solidaridad, el trabajo en grupo, entre otros. Dicho ambiente se traduce en situaciones donde los niños y las niñas se puedan manifestar, ser escuchados y satisfacer sus necesidades de exploración, en medio de relaciones que enlazan personas, entornos 
y actividades entre sí. La práctica cotidiana de esos valores tiene la posibilidad de transformar el actuar y pensar del niño y la niña, no solo dentro del contexto escolar, sino más allá.

Mediante el proceso de investigación se logró apreciar la importancia de propiciar un encuentro entre la familia y el centro infantil para proporcionar diversas oportunidades que influyen en el aprendizaje, por ejemplo los cuestionamientos, opiniones, intereses y experiencias. El vínculo que se logre realizar entre estas dos entidades posibilita y fomenta la expresión de afectos, además de que proporciona confianza básica, autoestima y seguridad en los niños y las niñas. En este sentido, las actividades observadas, de conciencia hacia el cuidado de la naturaleza, así como la inclusión de madres, padres y abuelos en el aula, resultan esperanzadoras.

El proceso de compromiso y transformación de las docentes con el proceso de esta propuesta metodológica, permitió responder a las necesidades que se evidenciaron a través de las triangulaciones, planteándose nuevos retos. Estos cambios requirieron la búsqueda continua de materiales, diálogo y sugerencias de cómo presentárselas a los niños y niñas, respondiendo así a un ambiente más significativo y estético.

Se está tan acostumbrado a guiarse bajo un modelo tradicional que lo que ocurre en las aulas es visualizado como una realidad absoluta. No se analiza ni se percibe lo que hay detrás de cada acción. Todo se ve como natural. Por lo tanto, se supone qué es lo que debe ocurrir. Dentro de estos supuestos se encuentran las rutinas, los planeamientos, las etapas de desarrollo, entre otros. Por eso se deja de lado decodificar el currículo oculto.

Es necesario contar con modelos propositivos que evidencien la práctica educativa, desde la acción- reflexión y la reflexión-acción. Se crea una conciencia crítica que permita mejorar la calidad de la educación.

El ecoanálisis es un proceso inacabado que debe ser continuo. Esto permite que la educación inicial replantee nuevos retos que le permitan mejorar continuamente y que además ofrezca un ambiente crítico que se base en los intereses y necesidades de la población infantil, porque promueve espacios interesantes para la exploración, el descubrimiento y la experimentación, a través de su propia experiencia de aprendizaje en el trabajo.

\section{REFERENCIAS}

Chavarría, M. C. (2003). Una educación inicial comprometida hacia la paz y el desarrollo sostenible. Revista Educación, 27(2), 45-66. 
Chavarría, M.C. (2006). Contraste entre los paradigmas holista y tradicional y sus implicaciones para el uso de términos y enfoques. (Documento presentado a la Comisión Interdisciplinaria a cargo del Proyecto interinstitucional de apoyo para la ampliación de la cobertura de atención integral a la niñez de 0 a 2 años de edad). Facultad de Medicina, Universidad de Costa Rica.

Chavarría, M.C., Obando, M. y Orozco, C. (2009). Ecoanálisis y decodificación de lo cotidiano en las prácticas formativas en los programas de atención a la niñez en los centros infantiles universitarios. (Informe Final de Investigación). Instituto de Investigaciones en Educación e Instituto de Investigaciones Psicológicas, Universidad de Costa Rica.

Chavarría, M. C. y Orozco, C. (2006). Ecoanálisis como puerta de entrada a la decodificación de lo cotidiano: Hacia una educación posible. Revista Electrónica “Actualidades Investigativas en Educación”, 6(3), 1-35.

Chavarría, M. C., Orozco, C., Chacón, Y. y Ovares, M. (2000). Aciertos y vicisitudes en la búsqueda de valores hacia la paz y el desarrollo sostenible: La decodificación de lo cotidiano como herramienta teórico-práctica. Revista Costarricense de Psicología, 16(31), 23-45.

Chavarría, M. C., Orozco, C., Chacón, Y., Ovares, M. y Obando, M. (2000). La formación del preescolar como cosmovisión: En busca de valores hacia la paz y el desarrollo sostenible. Revista Educación, 24(2), 115-132.

Chavarría, M. C., Orozco, C., Ovares, M., Obando, M. y Rivera, M. (2007). Problematización en torno a una educación inicial holista y crítica. (Avances de Investigación). Instituto de Investigaciones Psicológicas, Universidad de Costa Rica.

Chaves, A.L. (2001). La educación preescolar en el contexto nacional (1970-1998). Revista Electrónica “Actualidades Investigativas en Educación”, 1(2), 1-14.

Durán, M., Fernández, E., Hidalgo, S., Quintanilla, A. y Ramírez, E. (2010). Acercamiento crítico al holismo en la educación preescolar. (Seminario de Investigación de Licenciatura en Preescolar. Universidad de Costa Rica. Costa Rica.

Freire, P. (1970). Pedagogía del oprimido. México: Siglo XXI.

Moraes, C. (1999). El paradigma educativo emergente. Costa Rica: Centro de Innovación Educativa de la Fundación Omar Dengo. 
Orozco, C. (2005). El ecoanálisis como herramienta metodológica para el mejoramiento de un ambiente-situación en un centro preescolar. (Proyecto de Graduación de Maestría en Psicopedagogía). Universidad de La Salle, Costa Rica.

Osoro, J.M. y Meng, O. (2008). Escenarios para el análisis y la construcción de un modelo de educación infantil. Revista Iberoamericana de Educación, (47), 15-31.

Rodríguez, W.C. (2007). La investigación educativa en la América Latina: Cuatro desafíos y algunas ideas para enfrentarlos. Ponencia presentada en el Simposio del Primer Encuentro Internacional de Investigación Educativa: El Aporte de Freire y Vygotski. San José, Costa Rica.

Sandín, M.P. (2003). Investigación cualitativa en educación, fundamentos y tradiciones. España: McGraw-Hill.

\section{ACERCA DE LAS AUTORAS}

Cynthia Orozco Castro: Docente e investigadora. Sede de Occidente, Universidad de Costa Rica, San Ramón, Costa Rica. Correo electrónico: cynoroz@gmail.com

Maricel Durán Rodríguez: Docente de educación preescolar.

Evelyn Fernández Orozco: Docente de educación preescolar.

Sarita Hidalgo Arias: Docente de educación preescolar.

Annia Quintanilla Segura: Docente de educación preescolar.

Eva Ramírez Moya: Docente de educación preescolar. 